\title{
Application of Subatomic Thermodynamics to Astrophysics.
}

THE presidential address to the Mathematics and Physics Section of the thirteenth Indian Science Congress held at Bombay on January 4, by Prof. M. N. Saha, contains a résumé of the work of the last few years on the physical interpretation of stellar spectra. We are unable to find space to reproduce the whole address, but the following summary and extracts include some of the main subjects surveyed.

Since the foundations of astrophysics were laid by Kirchhoff in I859, the spectroscopic survey of the heavens has been carried on with great vigour by many workers, and the spectra of more than 200,000 stars have been classified at the Harvard College Observatory. In the interpretation of the spectra, however, two difficulties have arisen. First, there are several elements, common on the earth, which are not represented in the spectra of the stars; secondly, the elements represented in the stars are not all the same, but vary from one star to another in such a way that a continuous sequence of spectra can be arranged corresponding to a sequence of colour from blue-white through yellow to red. The character of the spectral change along the sequence is that in going from blue to red stars the spectral lines indicate a gradually decreasing excitation stimulus.

Lockyer long ago explained the phenomena by supposing that a spectrum was an indication of temperature, and that in the high temperature blue stars the elements were dissociated into simpler forms. This hypothesis offered a sufficient explanation of the facts, but was unwelcome on account of its revolutionary character. In recent years, however, this criticism has ceased to hold good, and in Prof. Saha's address an "attempt has been made to show how modern discoveries have led us to a complete justification of the main ideas of Lockyer."

When a gas is raised to a high temperature, the violence of the atomic collisions is conceived to result in the detachment of electrons from atoms. At any temperature a state of statistical equilibrium is reached, in which the rate of ionisation is equal to the rate of recombination. The gas then consists of ionised atoms, neutral atoms, and electrons, and at each recombination of an electron with an ionised atom, radiation is emitted. The fraction $(x)$ of dissociation can be calculated in terms of temperature $(T)$ and pressure $(P)$, and is found to be given by the equation :

$$
\log \frac{x^{2}}{I-x^{2}} \cdot P=-\frac{U}{2 \cdot 3 R T}+\frac{5}{2} \log T-6 \cdot 5=K,
$$

where $U$ is the ionisation potential of the atom and $R$ is the gas constant. The formula, as Russell has shown, needs modification when more than one element is present-a statement confirmed by both experiment and observation-and, further, represents only an approximation to the complexity of an actual case, since the various excited states of the atom have not been distinguished, but it is sufficiently exact to explain the main details of the spectral sequence.

From the observed positions in the sequence at which particular lines appear or disappear (which we may take to indicate the conditions under which the appropriate atoms begin or cease to be capable of emitting the respective lines), the above equation, combined with spectral data, gives a relation between the temperature and pressure in the stellar atmosphere. Assuming a likely value for the latter, the temperature can be estimated, and is found to agree fairly well with the photospheric temperatures mea- sured by direct experiment. This constitutes a justification for the view that ionisation in stellar atmospheres is a function of temperature and pressure. It has long been an unsettled question whether temperature alone can stimulate radiation, but recent experimental evidence indicates very definitely that it can do so. The theory under consideration not only provides for this, but also shows that the failure of earlier experiments was due to the choice of elements having high ionisation potentials, which, according to the equation, require high temperatures for ionisation or excitation. The implication that the degree of ionisation is increased by lowering the pressure is also borne out by experiment. The theory further explains the apparent absence of certain elements from the sun and stars by the argument that their chief spectrum lines under the conditions existing in the atmospheres are outside the range of observation.

Prof. Saha, to whom the theory in the above form is due, points to further evidence of thermal ionisation in the fact that the electrical conductivity of heated gases depends on their ionisation potentials. This at once follows from the theory if the conductivity is due to the presence of free electrons, for it must then be dependent on the percentage of ionisation, which is a function of the ionisation potential.

Later developments of the theory are mainly indebted to applications of subatomic thermodynamics to the problem. Following the lines of Ehrenfest's re-examination of Planck's thermodynamics, Fowler and Milne, Becker, Saha and Sur, have been the chief workers in this field.

"The scope of Fowler and Milne's work will be realised from the following brief summary: As mentioned above, when an original mass of calcium is heated, the products of decomposition are not only ionised calcium atoms and electrons, but also calcium atoms in the excited states, which, being unstable, give rise to the line spectrum of calcium. As the spectrum of calcium is rather complicated we shall take a simpler case, namely, hydrogen. The products of decomposition are then $\mathrm{H}^{+}$, electrons, $\mathrm{H}$-atoms in $2_{1}, 2_{2}$, $3_{1}, 3_{2} \ldots$ states, radiation $\nu=R\left(\frac{\mathrm{I}}{\mathrm{I}^{2}}-\frac{\mathrm{I}}{n^{2}}\right)$ constituting the Lyman series, the lines $\nu=R\left(\frac{\mathrm{I}}{2^{2}}-\frac{\mathrm{I}}{n^{2}}\right)$, constituting the Balmer Series, etc. ... In the simple theory sketched above, we neglected these intermediate orbits on the ground that even at the highest temperatures, their proportion calculated according to Maxwell's theorem is small.

By applying Ehrenfest's method (which has been improved on mathematical points), Fowler obtains the equation :

$$
\log \frac{x^{2}}{I-x^{2}} P=K-\log b(T)
$$

in place of the original equation. The function $b(T)$ represents the occurrence of the intermediate orbits.

$$
b(T)=g_{1}+g_{2} e^{-\frac{U_{2}}{k T}}+\ldots g_{n} e^{-\frac{U_{n}}{k T}}+\ldots .
$$

where $g_{n}$ is the intrinsic weight of the $n$th intermediate orbit, $U_{n}$ its energy. According to Bohr $U_{n}=R\left(\mathrm{I}-\frac{\mathrm{I}}{n^{2}}\right) h, \quad(R=$ Rydberg number $)$, and $g_{n}=$ $n(n+\mathrm{I})$ (according to Herzfeld and Epstein).

It may be mentioned here that $b(T)$ is divergent, and in order to get over this difficulty, Fowler thinks that $b(T)$ ought to be limited to a finite number of 
terms. He argues that as the $n$th orbit has a radius of $a_{0} n^{2}$, the series cannot be developed when the distance between two atoms is of this order, for the electron then passes into no-man's-land. As was pointed out in a note to NATURE, there is strong experimental evidence for believing that the development of higher orbits depends upon pressure (one has to think of Wood's method of getting higher lines of the Balmer series), and this is also supported by astrophysical data (see Miss C. Payne's "Stellar Atmospheres," page 48). Urey has recently shown that $b(T)$ becomes convergent when the finite volume of molecules is taken into account.

But the weak point in the argument is the assumption $g_{n}=n(n+\mathrm{I})$, and it seems to be unsound on physical grounds.

The conclusions which Fowler, and Fowler and Milne (Month. Not. R.A.S., vol. 83, page 403) have drawn are, however, largely independent of the form of $b(T)$, and are of the greatest importance. It will be recalled that many elements, for example, mercury, have to be detected by subordinate lines, while others like calcium can be detected by principal, as well as subordinate lines. Fowler and Milne discovered a very remarkable difference between the behaviour of the principal and subordinate lines in stellar sequence. They found that while principal lines are most intense at the lowest temperatures, and gradually weaken in the higher classes, the subordinate lines are at first faint, then increase in intensity, reach a maximum, and then die out. The explanation is as follows: At the lowest temperature, all atoms are in the lowest state. Subordinate terms come out only at higher temperatures. But when the temperature is very high, the atom begins to get ionised, and hence the proportion of atoms in the subordinate states begins to fall off. A maximum must have been reached in the interval.

When the above ideas are translated into mathematical language, and conditions for maximum concentration of subordinate levels applied, we get a relation connecting the electron pressure and the temperature in the reversing layer. In fact

$$
P_{e}=\frac{0.332}{b(T)} \cdot \frac{U_{r}+{ }_{2}^{5} R T}{U_{1}-U_{r}} T^{5 / 2} e^{-\frac{U_{1}}{k T} .} .
$$

For example, the Balmer-lines of hydrogen arise from the 2-orbit, and they reach their maximum, in the A-class. The temperature of these stars lies between $10,000^{\circ} \mathrm{K}$ to $12,000^{\circ} \mathrm{K}$. . Substituting these values in (I), we obtain $P_{e}=\mathrm{I} \cdot 3 \mathrm{I} \times 10^{-4}$ to $3.07 \times 10^{-3}$ atmospheres.

Fowler and Milne have thus found a very elegant method of determining the pressure. This is in itself a result of the first magnitude, for ionisation depends very largely on pressure, and after Einstein's discovery of the shift of lines towards the red by gravitational potential, the older values which were based on pressure-shift of lines have been rendered obsolete.

Values of $P_{\mathrm{e}}$ have been calculated from considerations of the maxima of several elements, and they invariably give a range of pressure varying from $\mathrm{IO}^{-3}$ to $10^{-5}$ atmospheres. These values recall the statement of Jewell; 'The whole mass in the reversing layer of the sun can be contained in a thimble.'. .

Prof. Milne has recently given an entirely new method of dealing with thermal ionisation of gases. In contradistinction to the thermodynamical method hitherto used, this method may be called the kinetic method. These two methods find their parallel even in the study of chemical equilibria. Let us represent a typical homogeneous equilibrium by the equation $m A+n B \longleftrightarrow p C+q D$.

Then according to the thermodynamical method we put

$$
m S_{A}+n S_{B}-p S_{C}-q S_{D}=\frac{U}{T},
$$

where $S$ 's are the entropies, and $U$ is the heat of reaction.

In the kinetic method, the rate of change from left to right depends on the number of times $m$ molecules of $A$ come into contact with $n$ molecules of $B$. The velocity of reaction $V_{A B}=K_{1} C_{A} C_{B}$, and $V_{O D}=K_{2} C_{C} C_{D}$. In the equilibrium case

$$
V_{A B}=V_{C D}, \text { hence } \frac{C_{A} C_{B}}{C_{C} \overline{C_{D}}}=\frac{K_{2}}{K_{1}}=\text { constant. }
$$

By using this method, Boltzmann gave a formula for molecular dissociation, which has the same form as that derived from thermodynamics.

Thermal ionisation of gases is only a particular form of chemical dissociation with the difference that the details of the mechanism are much better understood in the former case. Hence it is expected that the kinetic method would be very fruitful here.

Every kinetic method depends upon a closer treatment of the mechanism of reaction. The reaction in the present case is decomposition of $M$ into $M^{+}$and $e$ (electron), and this can take place in various ways. To any one of these processes there is an opposite process resulting in recombination of $M^{+}$and $e$ to $M$ A pair of such oppositely directed processes has been called by R. H. Fowler 'a unit mechanism.' Each one of these unit mechanisms, by itself and without the aid of any other process, would give us some fundamental laws of general validity. This is known as the "principle of detailed balancing."

The ionisation of $M$-atoms placed in a thermal enclosure can occur :

(a) As a result of collision of two $\mathrm{H}$ atoms; the rate of ionisation is then proportional to the square of pressure.

(b) As a result of absorption of radiation by $\mathrm{H}$ atoms, normal as well as excited.

Thus, according to Bohr's theory, radiation of frequency higher than $\nu=\frac{R}{\mathrm{I}^{2}}$, when falling on the normal hydrogen atom, would completely ionise it. If the electron is in the 2 -state, radiation shorter than $\nu=\frac{R}{2^{2}}$ would ionise the atom. Such a process of ionisation may be called photoelectric ionisation. This is true of every element, and in the case of alkalies it has been definitely proved that their vapours are ionised by radiation shorter than that corresponding to the limit of the principal series.

The reverse processes are :

$\left(a^{\prime}\right)$ Process $(a)$, ionisation by collision is radiationless. The reverse process of capture when $M^{+}$and $e$ come together must also be radiation-less. Hence when $M^{+}$and $e$ combine together, the energy set free must be carried away by a third body. Hence the reverse process to $(a)$ is a three body encounter between $M^{+}, e$, and $M$ (or $e$ ), the energy liberated being carried off by $M$. The unit mechanism $(a),\left(a^{\prime}\right)$ has been studied by R. H. Fowler in an extension of the ideas of Klein and Rosseland on the so-called collisions of the second type.

$\left(b^{\prime}\right)$, the reverse process to $(b)$, must naturally be the capture of an electron by the ion $M^{+}$, with liberation of radiant energy. But Milne finds that $(b)$ and $\left(b^{\prime}\right)$ together do not give the law of reaction isochore. So he postulates that electrons may be 
captured even under the influence of radiation. This last process is analogous to Einstein's 'Negative Einstrahlung ' or stimulated emission. Thus we may write-

Rate of photoelectric ionisation $=$ Rate of capture with emission + Rate of capture under the influence of radiation.

Compare this with Einstein's well-known method of deducing the law of black body radiation :

Rate of absorption of light $=$ Rate of spontaneous emission + Rate of emission under the influence of radiation.

With the aid of certain assumptions, Milne calculates the rates of free and stimulated captures and equates it to the number of photoelectric ejections. The equation may be used in two ways. It may be employed to evaluate the degree of ionisation, in which case some further assumptions are necessary. Conversely, assuming the thermodynamic formula for ionisation, it can be used for finding out the law of probability of electron capture. In this way Milne arrives at the conclusion previously reached by Eddington in his study of opacity of stars, namely, that the electron is captured only when it actually hits the nucleus. Probably a more rational way of expressing the result would be: Only one in $\mathrm{ro}^{5}$ collisions results in a capture.

Applied to hydrogen, calcium, and mercury, Milne's method furnishes values of absorption coefficients which are in general agreement with experimental values.

It is yet too early to predict how far Milne's method in its present form will be able to advance the theory of thermal ionisation. The great difficulty which is encountered in the development of these methods is due to the absence of any suitable quantum theory of absorption of lines. The present theories are a sort of half-hearted compromise with the old Lorentz theory which ascribes absorption to damping produced by collision."

\section{The Sea-Urchins of New Zealand.}

\section{By H. Farouhar, Wellington, N.Z.}

$\mathrm{T}$ $\mathrm{HE}$ following notes were written some time since, but were held back until Dr. Mortensen 'had published his zoogeographical notes on New Zealand echinoderms (Vidensk. Medd., Bd. 79, r925). They are now given here because the subject is approached from a different point of view ; namely, that of the New Zealand biological region; and also because I find myself obliged to differ from him on some points. For example, Dr. Mortensen believes that Wegener's hypothesis of continental displacement " gets support also from the study of the New Zealand Echinoderm fauna" ; while I am of the opinion that the evidence of our fauna and flora at large, including the littoral, is strongly against Wegener's hypothesis as regards Australia and New Zealand; and that it mainly agrees with the evidence of the micro-Lepidoptera as stated by Mr. E. Meyrick in NATURE of May 30, 1925. The more I have learnt of the New Zealand fauna and flora in general, and particularly of several small groups, the more I have been impressed by the great fundamental differences bètween the faunas and floras of the two regions; and, as Meyrick says, "The amount of community shown here is then no greater than might be expected if the conditions had always been as at present," etc. When we remember, moreover, that the New Zealand land-area was greatly elevated and extended far to the north-west in early Tertiary times, and, again, to a less extent perhaps, later, we may well be surprised that the affinities are not much stronger.

Reasons for the recognition of the New Zealand area as a primary zoological region were set forth in NATURE of January II, Igoo. Objections by Mr. Wallace were given in the issue of January I 8 , and support by Prof. Alfred Newton and Mr. Hedley in the issue of January 25, and April I9, respectively. In a letter to me, at that time, Prof. Newton wrote, "I have no doubt that the littoral marine fauna has also an exceedingly interesting story to tell "; and as the several groups of the littoral come to be worked and revised the more distinctly and clearly does this "interesting story" become known. In the preface to his "Manual of N.Z. Mollusca," 1880 , that keen and indefatigable observer, the late Capt. Hutton, said, "The better the fauna of New Zealand becomes known, the more prominently does it stand out distinct from that of any other country" ; and this is now being verified by all New Zealand naturalists. What makes the history of our plants and animals especially interesting and important is that this area is a biological region of primary rank (Holloway, Trans. N.Z. Inst., vol. 55, p. 67; Cockayne, “ New Zealand Plants and their Story," etc.), for here and here only the botanical region is coterminous or almost coterminous with the zoological region; and the evidence of the botany, zoology, geology, palæontology, the physical conditions of the land, and the form of the sea-bed all harmoniously contribute to the wonderful past history and present status and character of this remarkable region.

Of course the littoral marine fauna does not speak so clearly and forcibly as the land fauna, for species of some of the groups (hydroid zoophytes, mollusks, echinoderms, etc.) have recently been widely spread by oceanic currents, but the evidence, in the main, agrees with that of the terrestrial plants and animals.

The New Zealand sea-urchins have been recently revised and the number of known forms increased by Dr. Mortensen of the Copenhagen Museum (Vidensk. Medd., Bd. 73, I92I) ; and Dr. H. L. Clark of the Museum of Comparative Zoology has given an excellent classification of the group, based upon all the light of recent research, in his "Catalogue of the Recent Sea-urchins in the British Museum," I925. The littoral forms are those which inhabit the area lying between high-water mark and the hundredfathom line (the continental shelf). Large portions of this zone are entirely unknown, and many more species will by-and-by be added to our list, which at present consists of 22 forms :
I. Goniocidaris umbraculum, Hutton.
Austrocidaris benhami (Mortensen).
3. Cidaris sp. (Young), Mortensen. 4. Arcosoma thetidis (H. L. Clark) Amblypneustes ovum var. pach- ista, H. L. Clark.
6. Holopneustes inflatus, A. Agassiz. 7. Pseudechinus albocinctus (Hutton!.
8. Pseudechinus novcezealandia (Mortensen).
9. Psetidechinus huttoni, Benham.
10. Pseudechinus variegatus, Mort- ensen.
I1. Pseudechinus grossularius (Studer).

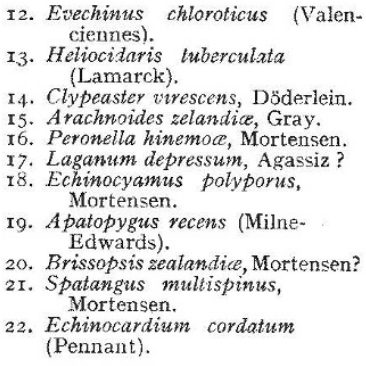

